\title{
Standing in the present, looking into the future-the 6th Joint Conference on Surgery and Academic Skills-precise treatment for tumors: medication and surgery
}

\author{
Andre Miao, Santeria Qiu \\ Editorial Office, AME Publishing Company \\ Correspondence to: Andre Miao, Santeria Qiu. Editorial Office, AME Publishing Company. Email: editor@thehbsn.org.
}

Submitted Oct 12, 2018. Accepted for publication Oct 12, 2018.

doi: $10.21037 /$ hbsn.2018.11.18

View this article at: http://dx.doi.org/10.21037/hbsn.2018.11.18

Throughout the ages, the autumn in Beijing has been praised for its beauty by poets and writers. It seems to have a unique, magic power that can attract every passerby. It was in such a wonderful setting that the 6th Joint Conference on Surgery and Academic Skills was successfully convened at the University of Chicago Center in Beijing. The conference was held from September 14-15, and jointly sponsored by the Peking Union Medical College Hospital, AME Publishing Company, Hepatobiliary Surgery and Nutrition (HBSN), the University of Chicago Global Surgery, the University of Chicago Department of Surgery, and the University of Chicago Center in Beijing. This year's conference mainly included five sessions: precision medicine, genomic therapy, modifying hosts and diseases, new insights into targeting malignancies, and finally, academic and publishing skills. The whole conference was aimed at examining both the science and art of providing precision medicine and surgery to patients with solid tumors. Dozens of experts and scholars from home and abroad attended this fabulous meeting and shared their research progress, along with their unique experience (Figure 1).

\section{Session I Precision medicine}

At the opening ceremony, as one of the conference cochairs, Prof. J. Michael Millis from the University of Chicago, vice chair for Global Surgery, extended his warm welcome and greetings to all speakers and members of the audience. The first session then began.

The first speaker was Dr. Alan Venook who is the Madden Family Distinguished Professor of Medical Oncology and Translational Research at the University of
California in San Francisco (UCSF). He made a keynote speech on the question "is there an optimal choice for managing colorectal cancer (CRC) liver metastases?” In his speech, he introduced the current status of CRC in the United States, the relationship between CRC and liver metastasis, and the methods involved in managing CRC and liver metastasis. He highlighted the importance of finding the mutation that causes CRC, because it directly determines which inhibitors should be used to solve the cancer metastasis problem. The doctor also mentioned that treatment of colorectal liver metastases requires multidisciplinary care, such as neoadjuvant therapy. $\mathrm{He}$ presented many graphs, images and other intuitive data in his presentation, vividly expounding his views to the audience (Figure 2).

Following this, Dr. Kai Wang, CEO of OrigiMed Inc., Shanghai, led a talk which focused on the analysis and application of precision medicine in patients with hepatobiliary cancers in China. His speech centered around the following three topics: (I) the development of molecular markers for prognosis of liver and gallbladder tumors, (II) the exploration and progress of targeted therapeutic markers in hepatobiliary tumors, and (III) the progress in immunotherapy and exploration of potential molecular markers. He concluded that given the innovations already achieved in cancer medicine, there is an impetus for more doctors to discover novel methods and uncover new areas in the world of cancer treatment.

Funda Meric-Bernstam, a professor in the Divisions of Cancer Medicine and Surgery at MD Anderson Cancer Center, then made a speech on precision medicine decision support. She introduced several key points for matching 

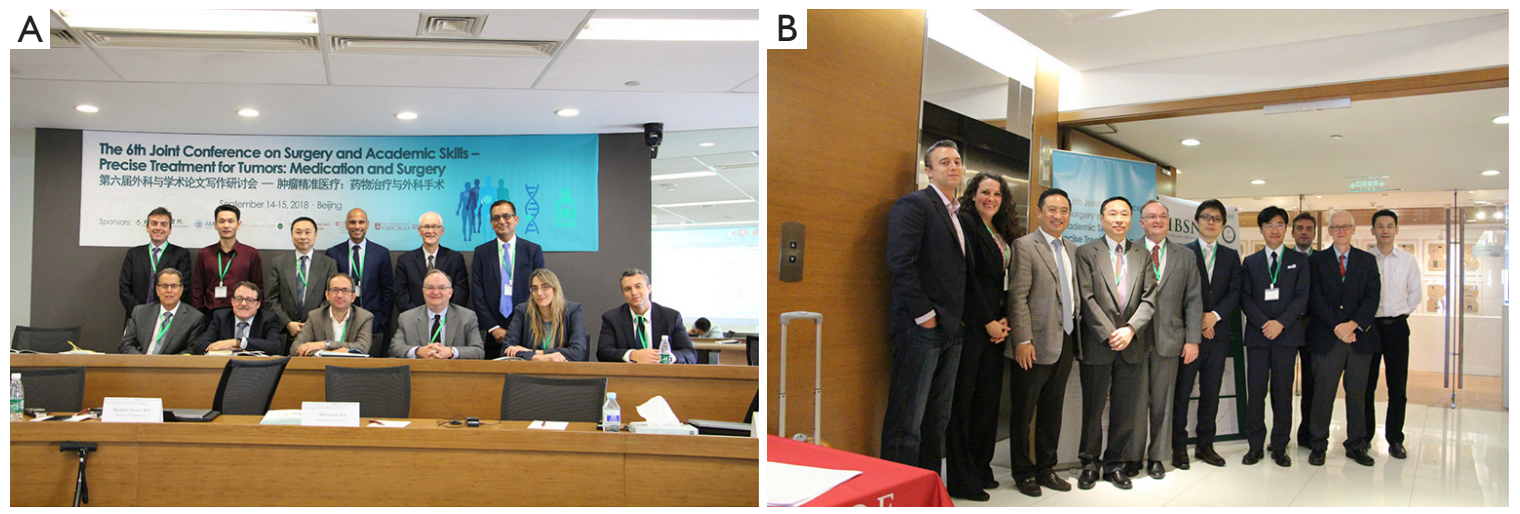

Figure 1 The bulk of invited speakers gathered at the conference.

accurate oncology decision support with clinical trials, which included, among other protocols, the optimal timing in molecular testing, the informed testing by physicians, and the screening of patients by clinical testers. To capture the core message of oncology decision-making in one sentence, one could say "give the right medicine to the right patient at the right time".

After this, Dr. Mustafa Hussain from Minimally Invasive Gastrointestinal and Bariatric Surgery in the University of Chicago Medical Center, described the multimodal localization techniques in minimally invasive surgery. In the beginning, he briefly explained the current understandings of tumor localization. He then noted that there are many techniques during surgery, including preoperative marking, intraoperative endoscopy, ultrasound and imageguided surgery—all which are responsible for improving, locating, and completing resection. This discussion was further elucidated for the audience by a rich collection of accompanying medical images and videos provided by Dr. Hussain in his presentation (Figure 3).

\section{Session II Genomic therapy}

Hosted by Prof. Simon C. Robson from Harvard Medical School and the Beth Israel Deaconess Medical Center, the second session highlighted genomic therapy. First, Milind Javle, a professor of medicine at the University of Texas MD Anderson Cancer Center in Houston, proposed the following points for targeted therapies in biliary cancer: (I) the use of somatic cell mutation spectrum in biliary cancer is feasible, and thus has clinical application value; (II) different gene change models are dependent on the location of the tumor (in or out of the liver); (III) the impact of the treatment and prognosis of biliary cancer requires more prospective studies.

The next speaker, Dr. Samuel J. Klempner, director of Precision Medicine and GI Oncology, shared his research about the benefits of genomic heterogeneity and translating molecular classification in treating gastric cancer. His talk focalized around the following ideas: (I) in most gastric cancers, heterogeneities exist both inside the same tumor and between different tumors; (II) subclonal drive affects the timing of treatment and drug resistance, which suggests that single biomarker detection is inadequate and genomic content is important; (III) clinicians should choose low frequency events to control disease progression in treatment; (IV) the increase of heterogeneity leads to multidrug resistance; (V) single-cell technology might provide more detailed information; (VI) perhaps more investigation into increasing heterogeneity is called for (Figure 4).

Dr. Mitchell C. Posner, physician-in-chief of the Comprehensive Cancer Center at the University of Chicago, made a speech titled "Tailoring Therapies for Gastro-Intestinal Malignancies". His speech unfolded from the seed of a key clinical question: "can we cure more cancer patients?" He then spoke about his research goal, which was to complete a comprehensive analysis of the molecular subtypes of clinical metastasis. Dr. Posner also suggested that molecular subtypes could complement clinical risk stratification, and he introduced the biological basis of potentially curable oligometastatic CRC.

On behalf of Dr. Haitao Zhao, who is a professor from the Department of Liver Surgery, at Peking Union Medical College Hospital, Dr. Jianzhen Lin, Haitao Zhao's student, gave the last speech of the first day. $\mathrm{He}$ 

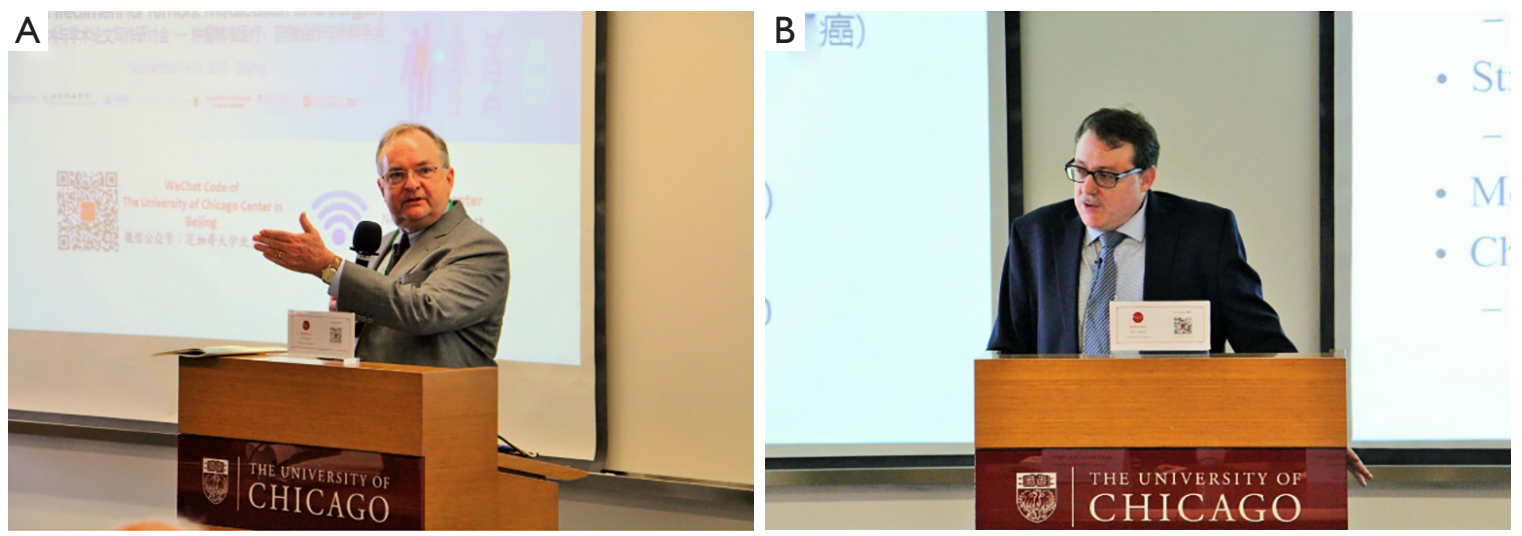

Figure 2 Prof. J. Michael Millis (A) and Dr. Alan Venook (B).
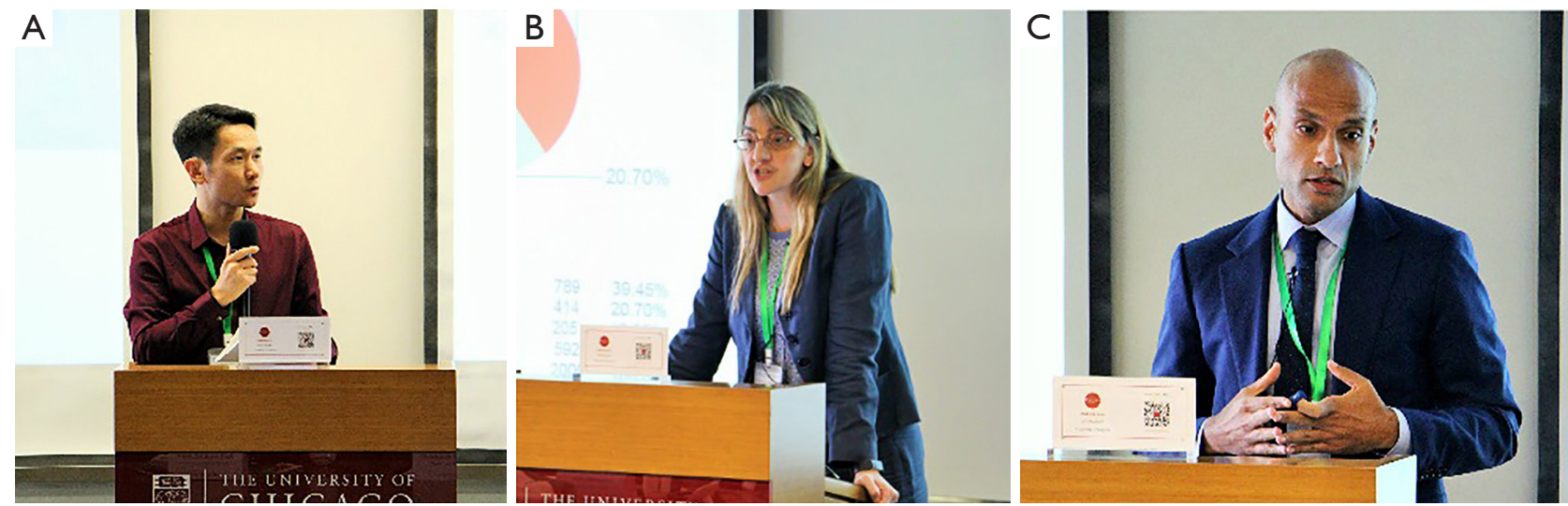

Figure 3 Dr. Kai Wang (A), Prof. Funda Meric-Bernstam (B) and Dr. Mustafa Hussain (C).
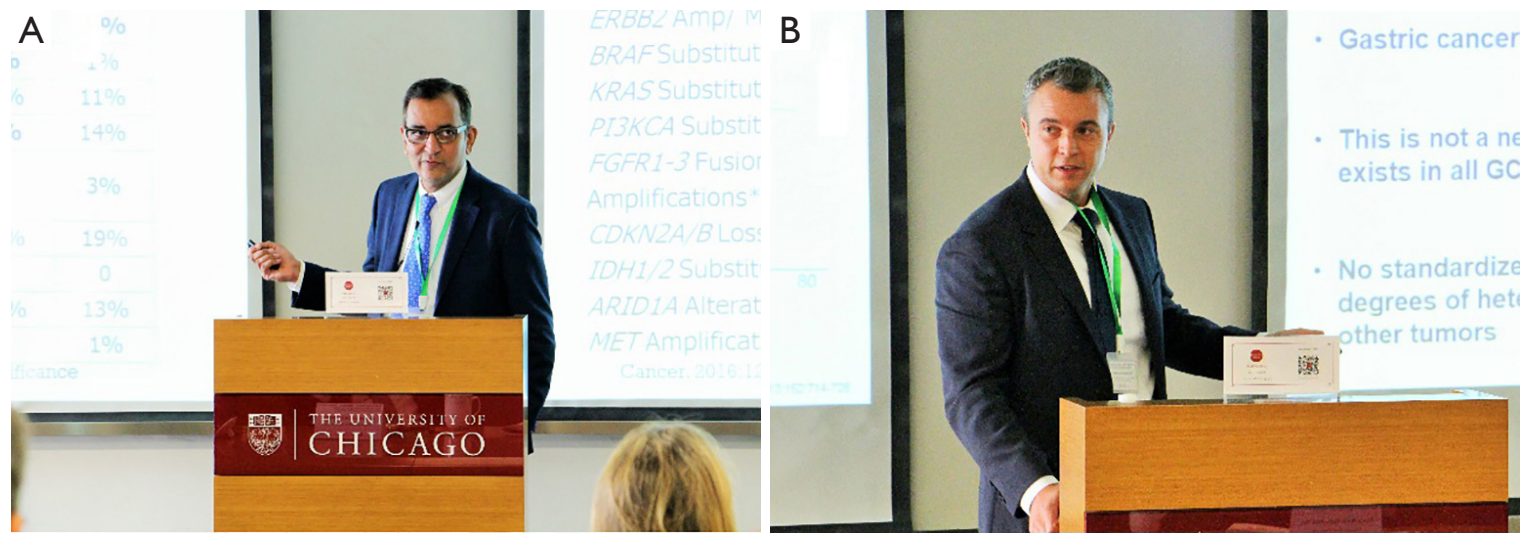

Figure 4 Prof. Milind Javle (A) and Dr. Samuel J. Klempner (B). 
discussed precision medicine and the immunotherapy of hepatobiliary malignant tumors. First, he gave a brief introduction to the epidemiology of hepatobiliary cancer in the world, and detailed the etiology and molecular classification of liver cancer in addition to the gene mutations and drug targets of biliary tract cancer. He then identified the antineoplastic drugs for the systematic treatment of HCC, such as sorafenib, lenvatinib, regorafenib, etc. Dr. Lin cited several examples to demonstrate the accuracy of precision medicine and immunotherapy, and the results are encouraging (Figure 5).

\section{Session III Modifying hosts and diseases}

On the second day, the conference started with a keynote speech via Skype by Prof. Matthew L. Meyerson from the Department of Pathology in Harvard Medical School. The topic was genomic alterations in human cancers, and the professor touched upon the following areas: (I) the cancer genome; (II) the question of identifying the most important type of gene mutations; (III) the patterns of gene mutation; (IV) the general characteristics of the lung cancer genome and targeted therapy for the lung adenocarcinoma gene; (V) the use of a lung cancer sequence for diagnosis; (VI) the non-coding genomic alterations in lung and prostate cancer.

Subsequently, Dr. Ashley Williamson, a surgical resident from the University of Chicago, delivered a speech on modifying commensal microbiota, which are novel modifiable risk factors for colon cancer. She first introduced the current situation of colon cancer in China and the United States, the incidence of colon cancer and the relationship between incidence and dietary patterns. She noted that diet, microorganisms and metabolism are closely related to colon cancer, and that the mechanism of dietary factors is mediated by colon microorganisms. Dr. Williamson finally speculated that, after operation, Enterococcus faecalis and abundant microflora can drive the metastasis and recurrence potential of colon cancerspeculation which is a new target of intervention.

After this, Prof. Jian Zhou from Zhongshan Hospital, at Fudan University discussed liver transplantation for liver cancer in China. He first explained that the outcome of liver transplantation for hepatocellular carcinoma (HCC) is determined by cancer-related and unrelated events. $\mathrm{He}$ then outlined how the treatment for the hepatitis $\mathrm{C}$ virus infection reduces non-cancer events in liver transplant recipients, and so reducing HCC-related deaths may be a viable end-point. Finally, Prof. Zhou explicated how in his research he conducted a competitive risk analysis to assess factors associated with the survival of HCC patients and established a prognostic model based on the characteristics of HCC patients before liver transplantation (Figure 6).

Following Prof. Zhou's speech, a talk was given by Dr. Toshimi Kaido from the Department of Surgery at Kyoto University. In his presentation, he emphasized the significance of preoperative sarcopenia in liver surgery. Additionally, he pointed out that liver surgery is closely related to myopathy and visceral adipose tissue.

Dr. Nobuhisa Akamatsu, from the Department of Surgery, at the University of Tokyo, also focused his research on liver transplantation for HCC. He indicated that Milan's criteria for measuring and defining early HCC are slightly outdated in relation to the current state of HCC development, and thus more apt criteria need to be designated (Figure 7).

\section{Session IV New insights into targeting malignancies}

The fourth session started with Dr. Matteo Donadon's speech. As an assistant professor of surgery from Humanitas University in Italy, Dr. Donadon talked about decoding the clinical heterogeneity of colorectal liver metastases by characterization of tumor-associated immune cells. He asserted in his speech that the immune system plays an important role in patients with liver metastases from CRC. He went on to explain how tumor-associated macrophages look promising for the immunotherapy of CRC patients with liver metastases. He rounded off his talk by stating that because the hypothesis of tumor-associated macrophages having different subsets has been confirmed, it means that metabolic and proteomic analyses can be carried out via mass spectrometry techniques.

The next speaker, Prof. Simon C. Robson, shared his study on novel purinergic check point inhibitor targets in malignancy. He found that purines, which regulate immune cells, can inhibit CD39 by modulating the signaling pathway, which means that they can achieve significant immune response in cancer models. He noted that targeting CD39 has potential therapeutic value in the use of checkpoint inhibitors in human diseases, and that laboratories are also evaluating the efficacy of anti-CD39 monoclonal antibodies and other forms of treatment in cancer.

The last speaker of this session was Dr. Fabirizio Panaro, a professor of surgery from Montpellier University in 

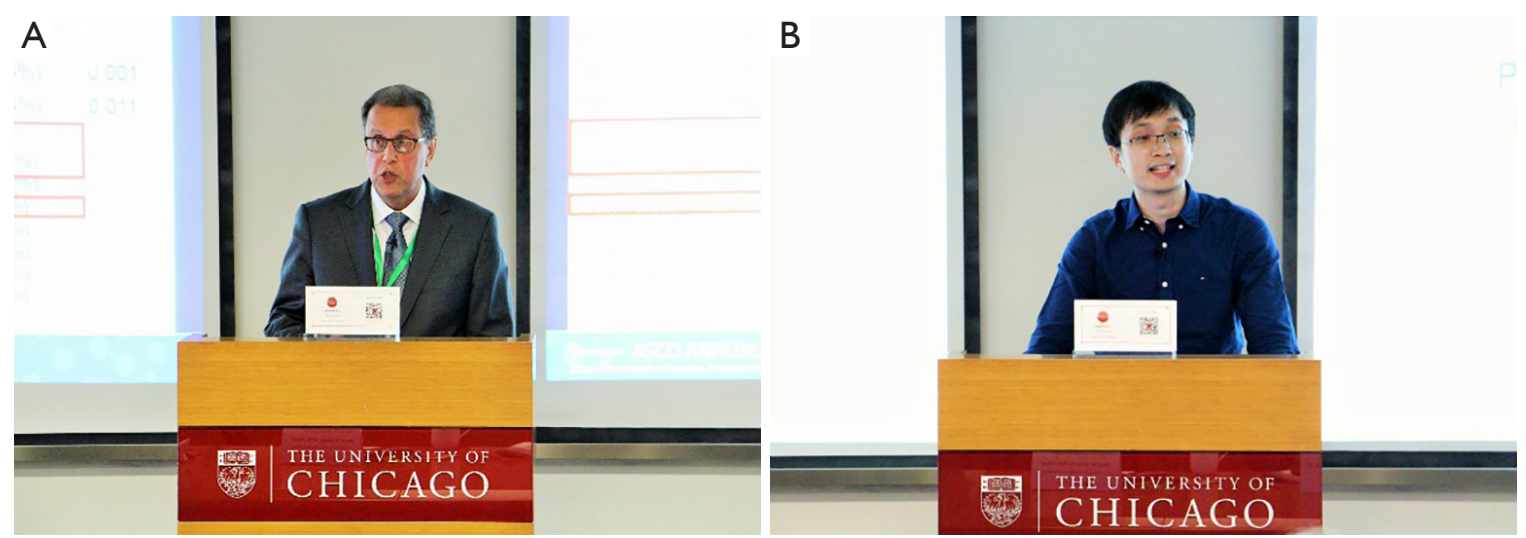

Figure 5 Dr. Mitchell C. Posner (A) and Dr. Jianzhen Lin (B).
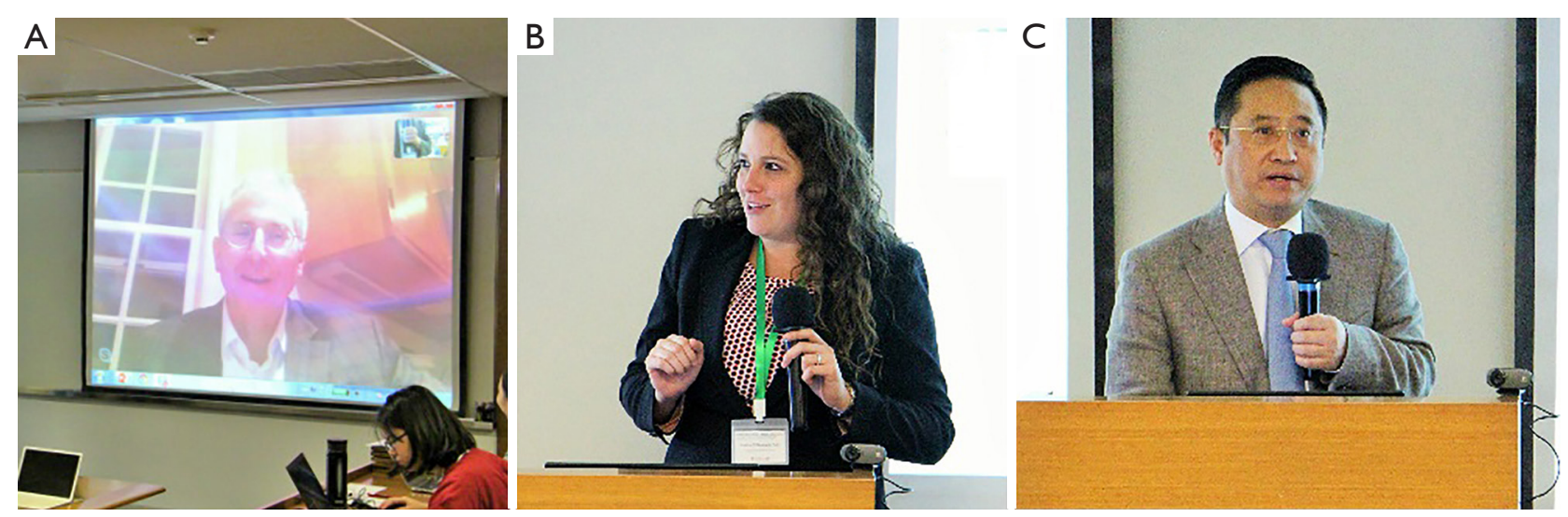

Figure 6 Prof. Matthew L. Meyerson (A), Dr. Ashley Williamson (B) and Prof. Jian Zhou (C).
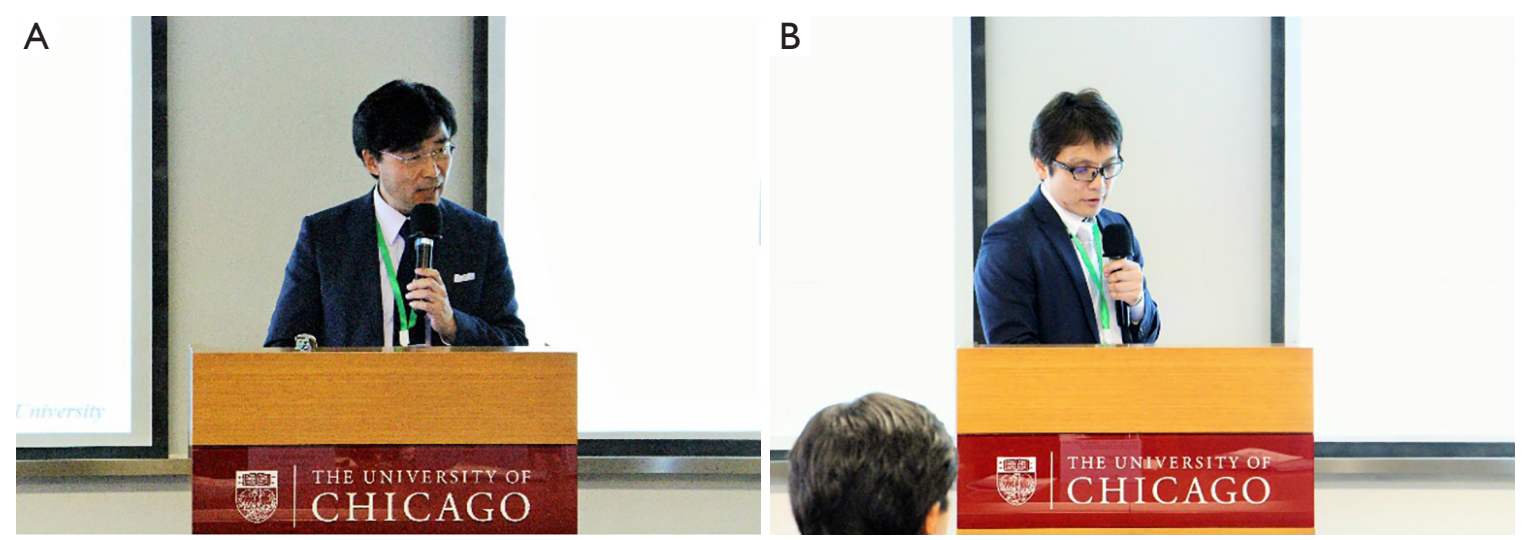

Figure 7 Dr. Toshimi Kaido (A) and Dr. Nobuhisa Akamatsu (B). 

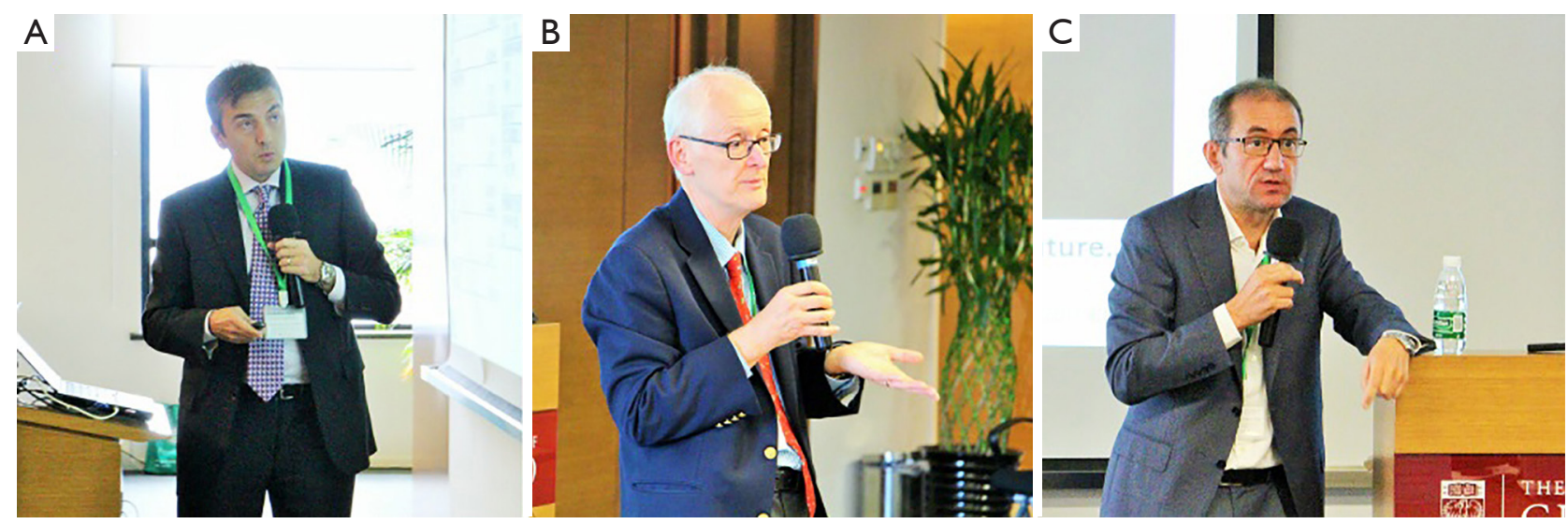

Figure 8 Dr. Matteo Donadon (A), Prof. Simon C. Robson (B) and Dr. Fabirizio Panaro (C).

France. He made a speech on the role of the indocyanine green (ICG) fluorescence during a hepato-biliary surgery. First, he introduced ICG, a fluorescent dye widely used in fluorescence imaging of hepatobiliary surgery, and briefly brought up how to use ICG for intraoperative imaging. Next, Dr. Panaro explained the principle of using ICG tumor imaging and the role of ICG in liver transplantation (Figure 8).

\section{Session V Academic and publishing skills}

The last session of the conference revolved around the principles in scientific publications and academic writing skills. Dr. Ashley Williamson introduced the American general surgery research scholarship and mentioned that in the United States, surgeons usually pay more attention to research than other doctors. She particularly emphasized the importance of laboratory time during residency.

Dr. Toshimi Kaido then presented his experience on how to make a good presentation and write academic articles. He pointed out that a presentation is called a "presentation" because it is the "present" that the speaker brings to the audience. In fact, there are skills in making slides, writing summaries, and performing on stage. At the end of his speech, he told the audience, "if mind changes, then life changes".

Next, Dr. Mitchell C. Posner explained the importance of mentorship to academic surgeons. To make his speech better understood by the audience, he demonstrated the mentorship model from four aspects: environment, curriculum, role models and wise advice. The discussion of problems in the surgical field mentioned by Dr. Posner resounded with the audience. After discussing how to be both a successful mentor and an outstanding student, he gave the audience some prudent and practical suggestions.

To conclude the conference, Dr. Nobuhisa Akamatsu, from Japan, gave a talk on how to write and publish English papers from a non-English speaker's point of view. He briefly introduced how he published his theses both as the first author or the corresponding author. Next, he showed several pie charts to illustrate how almost all the papers received will be revised to a certain extent before they are successfully published. He concluded that a never-give-up belief is important for an academic to get a paper published; once your manuscript is rejected, revise it and make another contribution right away.

Although the conference only lasted a day and a half, the atmosphere was markedly congenial and lively. Nearly every speaker's speech aroused heated discussion, as participants were deeply involved in the proceedings and would not relinquish a chance to put forward their questions for their colleagues' consideration. (Figure 9).

\section{Standing in present, looking into the future}

To echo a memorable sentiment shared by Dr. Posner in his speech-"what a doctor pursues in his lifetime is simply to save one more patient"-it is only the diligence and effort of the doctors of today, that promises the health and safety of the patients of tomorrow.

Here we give our sincere respect to every industrious doctor and extend our heartfelt gratitude to all the speakers and organizers for their considerable support and contributions to this conference. As a series meetings, this 


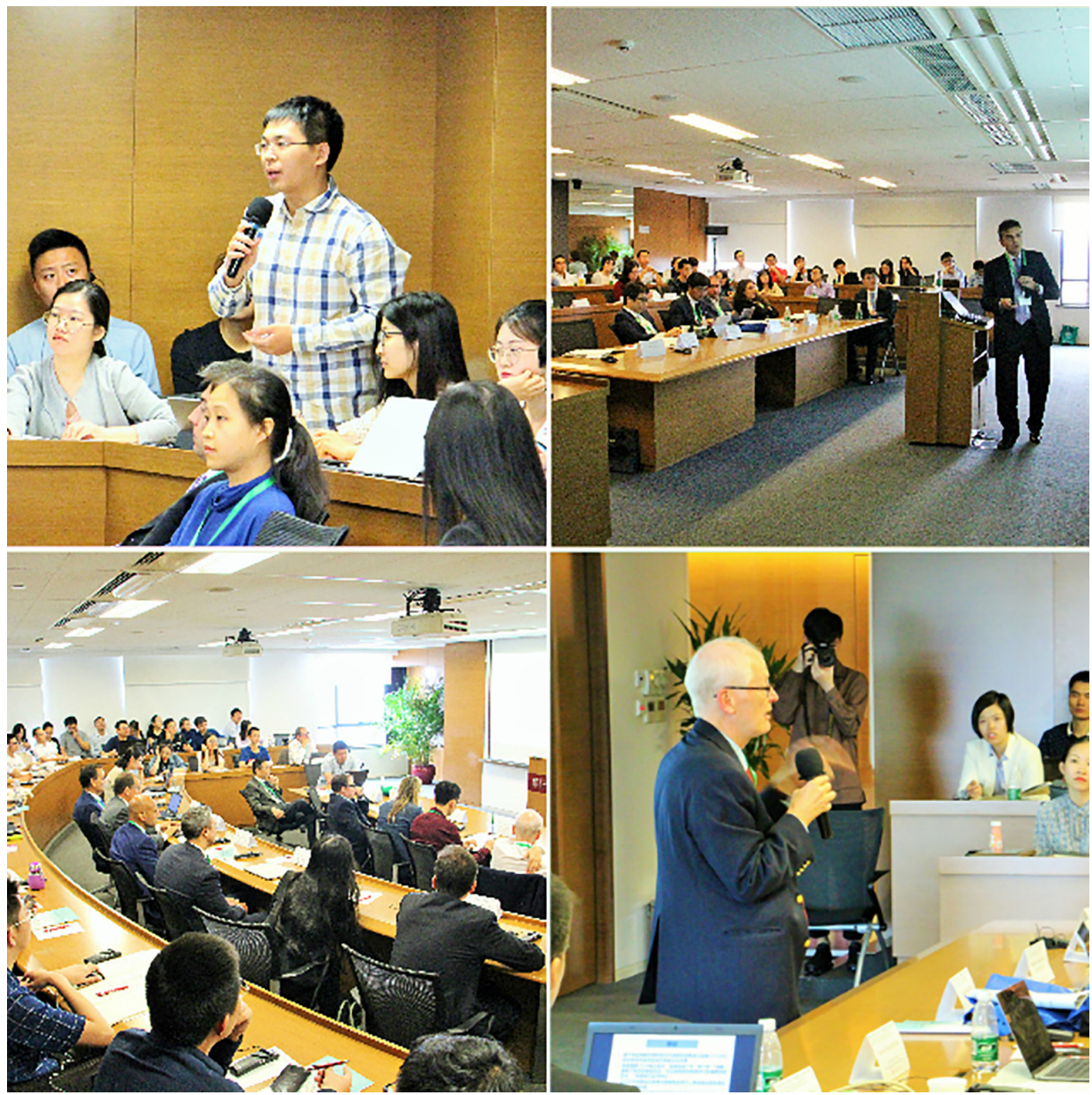

Figure 9 Discussion among experts and audience members.

conference will continue and be held in 2019. We warmly welcome your participation. You may contact us via the $H B S N$ journal email. We look forward to our next gathering in Beijing!

\section{Acknowledgements}

We would like to thank Silvia L. Zhou and Elva Zheng, editors of $H B S N$, for their guidance and for polishing this newsletter.

\section{Footnote}

Conflicts of Interest: The authors have no conflicts of interest to declare.

(English Language Editor: John Ayric Gray, AME Publishing Company)
Cite this article as: Miao A, Qiu S. Standing in the present, looking into the future-the 6th Joint Conference on Surgery and Academic Skills—precise treatment for tumors: medication and surgery. HepatoBiliary Surg Nutr 2018;7(6):508-514. doi: 10.21037/hbsn.2018.11.18 